\title{
On the Irreversibility in Mechanical Systems Using a New Macroscopic Energy Structure Modeling
}

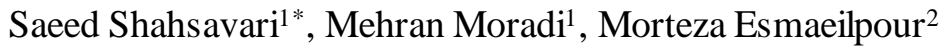 \\ ${ }^{1}$ Department of Mechanical Engineering, Isfahan University of Technology \\ Isfahan, Iran \\ ${ }^{2}$ Department of Civil Engineering, Geo and Environmental Sciences, Karlsruhe Institute of Technology \\ Kralsruhe, Germany \\ ${ }^{*}$ Corresponding author's email: s.shahsavari [AT] me.iut.ac.ir
}

\begin{abstract}
This paper presents a macroscopic applied innovate modeling to study the performance effects of the second law of thermodynamics on the mechanical systems. To investigate the irreversibility in mechanical systems, the energy structure of the system can be studied. Some energy components relate to the reversible processes and remaining relate to the irreversible process. Exiting models are based on the studying sub structures and therefore, need a large volume of the calculations. In this paper, at first, using a macroscopic quasi-statistical approach, a new energy structure equation is extracted and by examining it's variation in the different paths, the irreversible components as well as their structures are studied. Using the kinematic theories of dissipated energy, it can be concluded that the extracted equations have the same base as the different formulations of the second law of thermodynamics. Finally, as a mechanical system example with the possibility of irreversibility in the possible performed processes, the extracted equations are developed for viscoelasticityproblems. And also the matching of the results with expected results is shown.
\end{abstract}

Keywords---- Mechanical Systems; Energy Structure equation; Irreversibility; work bound; Viscoelasticity

\section{INTRODUCTION}

Irreversibility in one of the most important concepts from the perspective of all branches of science that has been extracted in the different formulations of the second law of thermodynamics $[1,2]$. Entropy is the most important quantity used to formulating of the second law and base on this law, entropy will be generated in all performed processes [2].

Equations (1) and (2) are two mains formulations of the second law:

$\oint \frac{\delta Q}{T} \leq 0$

$d s_{\text {gen }} \geq 0$

That $\delta Q$ is the heat exchanged at temperature $T$ and $s_{g e n}$ is the generated entropy.

In fact, second law states that always some of the useful energy exchanges to the thermal energy.

Based on the second law of thermodynamics, when some energy is applied to the system, the work done by system will has upper bound. Carnot has studied the upper bound in thermodynamic cycles [1]. Carnot's equation depends on the hot and cold temperature in considered Carnot's cycle only [2].

Also, investigating the effects of the second law on the performance of the mechanical systems may be very important in their design. Due to the second law, it can be concluded that mechanical systems (or dynamically loaded bodies) have upper bound to the work done by them [3]. The work bound for these systems must be studied using the relevant deformation theories and models. Also, exiting as well as value of the upper work bound in systems for multichannel queues has been investigated [4].

Also, from the other point of view work bound in mechanical systems can be studied. One of these approach is investigation from the pers pective of the free energy of the system and considered performed process [5]. Free energy is a part of the energy that can be used in the performed processes. The upper and lower bonds for free energy change can be using the second law formulation.

Discussion on the using of the second law to determine the work bound can be expanded by other examples. For example, work bounds cyclically loaded creeping structures can be presented [6]. In this case as well as similar cases, the sub- 
structures of the system are investigated [7-10]. Studying at the molecular level need using the molecular s tructure modeling as well as deformation theories.

One of the most important mechanical properties of materials that can be effective on the loss of the useful energy, is vis coelasticity. At non-zero energy applying rate, these materials, always experience irreversible process because of the dis sipated energy [11]. Therefore, due to the second law, these mechanical systems have the work bound. Dis sipation energy process can have different bases from the pers pective of the sub-structures [12-15]. In fact, their molecular structures have the main role in the relevant analysis.

The dis sipation energy can be studied using studying the dynamical on the sub-structures [16-19]. Some of the most important reas ons of the irreversibility in physical systems are: friction of solids; imperfect fluidity of fluids; imperfect elas ticity of solids; inequalities of temperature and consequent conduction of heat produced by stresses in solids and fluids; imperfect magnetic retentiveness; residual electric polarization of dielectrics; generation of heat by electric currents induced by motion; diffusion of fluids, solution of solids in fluids and other chemical changes and absorption of radiant heat and light.

In this paper, at first, using a new macroscopic quasi-statis tical approach to the physical processes, the effects of the second law on the energy structure are studied. Then, based on the macroscopic energy components of the systemas well as using the independent and dependent components concept, an energy structure is extracted. Also, using the examining of the variation of the energy structure equation in different paths, the irreversibility is studied and also, based on the energy components of the systemis modeled. Finally, as a mechanical systemexample, viscoelasticity problems are investigated and extracted equations are developed for these problems.

\section{MACROSCOPIC QUASI-STATISTICAL MODELING TO THE MECHANICAL PROCESSES}

If the same amount of energy is applied to the systemin different conditions, different energy components may be activated [16-18]. When energy is applied to the systemin a quasi-static path (Which is a path that the rateof applying energy can be considered zero to it), the performed process is known as a reversible process [19], and also, the activated components can be known as reversible components. These components (that can be named as independent components), also will be activated in other conditions of energy applying to the system(from the point of view of the energy applying rate). Finally, among activated components, some of them will be dependent on the independent components.

In general (non-zero applying energy rate), some secondary components may be activated that are dependent on the applying energy rate (or rate of the independent components) [16]. Therefore, it can be concluded that equation (3) can be considered as the energy structure equation of the system:

$U_{T}=\left(u_{1}+u_{2}+\cdots+u_{m}\right)+\left[g_{1}+\cdots+g_{k}\right]+\left[h_{1}+\cdots+h_{n}\right]+U_{T_{0}}$

Where:

$g_{j}=g_{j}\left(u_{1}, u_{2}, \ldots, u_{m}\right)$

$h_{p}=h_{p}\left(\dot{u}_{1}, \ldots, \dot{u}_{m}\right)$

The functions $g_{j}$ represent the value-dependent components of the independent components, while the functions $h_{p}$ show the components which are dependent on the rate of the independent components. Also $U_{T_{0}}$ represents the sum of all the components that did not participate in the performed process (in fact, this termcan be not considered in equation (3), because it does not change in performed process).

Equation (3) has the same base as the second law of thermodynamic. Based on the different formulations of the second law, always some of the available work will be converted to thermal energy. As we know that the thermal energy is dependent on the kinematic of sub-structures, while other forms of energy depending on the place and displacement of the sub-structures [20].

\section{IRREVERSIBILITY AND ITS STRUCTURE}

To investigate the irreversibility, it is assumed that the same amount of energy is applied to the system in different conditions. The quasi-static (zero rates of energy applying) path is considered as a reference path and the variation between this path and general paths (non-zero rate of energy applying) is calculated.

Therefore, using the energy structure equation when the same energy is applied to the system, equation (6) can be written: 
$\sum_{i=1}^{m}\left[\left(1+\sum_{j=1}^{k}\left(\frac{\partial g_{j}}{\partial u_{i}}\right)\right)\left(\delta u_{i}-\delta u_{i}^{\prime}\right)\right]=-\sum_{i=1}^{m}\left[\left(\sum_{p=1}^{n}\left(\frac{\partial h_{p}}{\partial \dot{u}_{i}}\right)\right) \delta \dot{u}_{i}\right]$

Where $\delta u_{i}$ is the variation of the $u_{i}$ when $\dot{u}_{i} \neq 0$ as the value of energy $\delta U_{T}$ is given to the systemand $\delta u_{i}^{\prime}$ is the variation of the $u_{i}$ when $\dot{u}_{i} \cong 0$ as the value of energy $\delta U_{T}$ is given to the system.

Equation (6) als o can be rewritten as follows:

$\left(1+\sum_{j=1}^{k}\left(\frac{\partial g_{j}}{\partial u_{i}}\right)\right)\left(\delta u_{i}-\delta u_{i}^{\prime}\right)=-\left(\sum_{p=1}^{n}\left(\frac{\partial h_{p}}{\partial \dot{u}_{i}}\right)\right) \delta \dot{u}_{i}$

Therefore, the variation between general and quasi-static paths is equal to $\left[-\left(\sum_{p=1}^{n}\left(\frac{\partial h_{p}}{\partial \dot{u}_{i}}\right)\right) \delta \dot{u}_{i}\right]$, as shown in figure 1 :

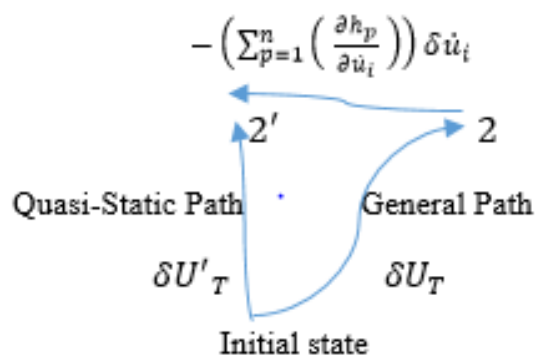

Figure 1. The variation between general and quasi-static paths

Therefore, based on the second law of thermodynamic, the term $\left[\left(\sum_{p=1}^{n}\left(\frac{\partial h_{p}}{\partial \dot{u}_{i}}\right)\right) \delta \dot{u}_{i}\right]$ must have the same sign with the applied energy $\delta U_{T}$. So it can be acriterion to investigate the irreversibility in the performed process. And also, by definition $\varphi$ as follows:

$\varphi=\frac{\left(\sum_{p=1}^{n}\left(\frac{\partial h_{p}}{\partial \dot{u}_{i}}\right)\right) \delta \dot{u}_{i}}{\delta U_{T}}$

It will be concluded that always $\varphi \geq 0$ (for all pos sible performed processes).

As expected, this quantity ( $\varphi \geq 0)$, for a particular mechanical system depends on the amount of applied energy to the systemand its applying conditions. Also, from the kinetic theory of dis sipated energy, it can be concluded that re lation (8) has the same baseas the second law of thermodynamics [20].

\section{LINEAR VISCOELASTICITY}

In this part, the presented equations are developed to the linear vis coelasticity problems, as a configurationally mechanical systemexample with the possibility of irreversibility in the performed process. The energy structure for generalized Kelvin and Maxwell models, as two main bas ic viscoelasticity models, is presented, and als o matching the results with expected results is shown.

\subsection{Generalized kelvin element}

Figure 2 shows the generalized Kelvin elements viscoelasticity model [21]:

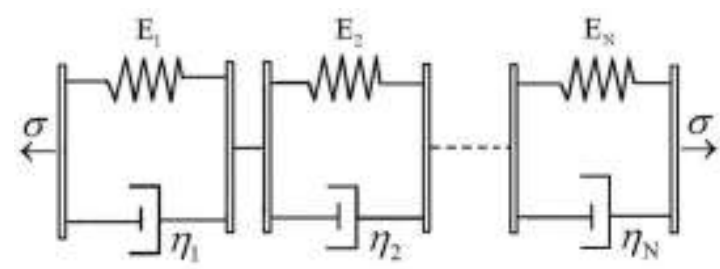

Figure 2. Generalized kelvin element

For this model, the energy structure can be rewritten as follows:

$U_{T}=u_{e 1}+u_{e 2}+\cdots+u_{e N}+c_{e 1} \dot{u}_{e 1}+c_{e 2} \dot{u}_{e 2}+\cdots+c_{e N} \dot{u}_{e N}+$ constant

Where $u_{e i}$ is the potentialenergy of $E_{i}, \dot{u}_{e i}$ is the rate of $u_{e i}$ and the coefficient $c_{e i}$ can be calculated as $\frac{\eta_{i}}{E_{i}}$.

To verify the equation (9), the following argument can be made: 
For the Kelvin model, the potential and dis sipated energy can be calculated from $\frac{\partial u_{e i}}{\partial \varepsilon_{i}}=E_{i} \varepsilon_{i}$ and $\frac{\partial u_{d i}}{\partial \varepsilon_{i}}=\eta_{i} \dot{\varepsilon}_{l}$, that $\varepsilon_{i}$ is the strain. Therefore it can be concluded that $u_{d i}=\frac{\eta_{i}}{E_{i}} \dot{u}_{e i}+f(t)$, where $f(t)$ is a function of time-related to the initial condition.

\subsection{Generalized Maxwellelement}

Figure 3 shows the generalized Kelvin elements viscoelasticity model [12]:

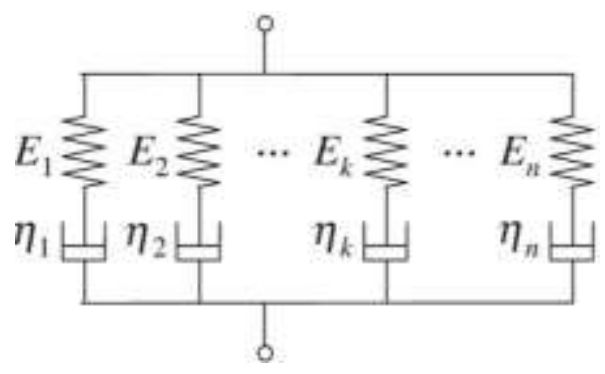

Figure 5. Generalized Maxwell element

For this model, the energy structure can be rewritten as follows:

$U_{T}=u_{d 1}+u_{d 2}+\cdots+u_{d N}+c_{d 1} \dot{u}_{d 1}+c_{d 2} \dot{u}_{d 2}+\cdots+c_{d N} \dot{u}_{d N}+$ constant

Where $u_{d i}$ is the dis sipated energy of $\eta_{i}, \dot{u}_{d i}$ is the rate of $u_{d i}$ and the coefficient $c_{d i}$ can be calculated as $\frac{\eta_{i}}{2 E_{i}}$.

To verify the equation (10), the following argument can be made:

For the Maxwell model, the potential and dis sipated energy can be calculated from $\frac{\partial u_{d i}}{\partial \varepsilon^{O V}{ }_{i}}=\eta \varepsilon^{O V}{ }_{i}=E_{i} \varepsilon^{e}{ }_{i}=\frac{\partial u_{e}}{\partial \varepsilon^{e}}$, that $\varepsilon^{O V}{ }_{i}$ is the strain of $\eta_{i}$ and $\varepsilon_{i}^{e}$ is the strain of $E_{i}$. Therefore it can be concluded that $u_{e i}=\frac{\eta_{i}}{2 E_{i}} \dot{u}_{d i}$.

\section{CONCLUSIONS}

To investigate the performance effects of the second law of thermodynamics on the mechanical systems, innovatively their macroscopic energy components can be studied from the pers pective of the energy structure. Equation (3) is considered as the energy structure equation to the system. When a particular process is performed, an energy structure equation is formed. Also, based on theenergy applying conditions, the independent and dependent components can be understood.

To investigate the irreversibility, it is assumed that the same amount of energy is applied to the system in different conditions, and the variation of the energy structure is studied. A quasi-static path is used as a reference path and the variation between this path with general paths is examined. Using this approach, equation (8) is extracted that introduces a positive quantity $(\varphi \geq 0)$ for performed processes. This quantity can be considered as a criterion for investigating the irreversibility during performed processes. As can be concluded, for a particular mechanical system, this quantity depends on the applying energy conditions to the system.

One of the most important results of this paper, due to the equation (3) and (8), is it that the irreversibility will be occured because of the independent components activated in performed process. In fact, when the conditions of energy applying as independent components exist in the energy structure of the system, the performed process is an irreversible process.

As viscoelastic materials can be known as materials with dissipated energy and irreversibility effects, in part 4, using the presented approach, viscoelasticity problems were investigated. Also the energy structure to the generalized Kelvin and Maxwell models (equations (9) and (10)), as two main basic viscoelasticity models, were presented.

\section{REFERENCES}

[1] Erlichson, Herman. "Sadi Carnot,Founder of the Second Law of Thermodynamics'." European journal ofphysics 20.3 (1999): 183.

[2] Sheehan, D. P. "The second law of thermodynamics: Foundations and status." Foundations of Physics 37.12 (2007): $1653-1658$.

[3] Ponter, A. R. S. "General dis placement and work bounds for dynamically loaded bodies." Journal of the Mechanics and Physics of Solids 23.2(1975): 151-163. 
[4] Wolff, Ronald W. "Upper bounds on work in system for multichannel queues." Journal of applied probability 24.2 (1987): 547-551.

[5] Reinhardt, WilliamP., and JohnE. Hunter III. "Variational path optimization and upper and lower bounds to free energy changes via finite time minimization of external work." The Journal of chemical physics 97.2(1992): 1599-1601.

[6] Ponter, Alan RS, and Jeffrey John Williams. "Work bounds and associated deformation of cyclically loaded creeping structures." (1973): 921-927.

[7] WILLIAMS, JJ. "Work Bounds and Associated Deformation of Cyclically Loaded Creeping Structures."

[81 Siirtola, Antti. "Bounds: from parameterised to finite-state verification." 2011 Eleventh International Conference on Application of Concurrency to System Design. IEEE, 2011.

[9] Danes, Florin, and Bertrand Garnier. "Effective conductivity bounds by inserting adiabatic or isothermal surfaces." International iournal of heat and mass transfer 54.15-16 (2011): 3523-3535.

[10] Carter, Peter. "Analysis of cyclic creep and rupture. Part 1: Bounding theorems and cyclic reference stres ses." International journal of pressure vessels and piping 82.1 (2005): 15-26.

[11] Layton, W. J. "Energy dissipation bounds for hear flows for a model in large eddy simulation." Mathematical and Computer Modelling 35.13 (2002): 1445-1451.

[12] Srivastava, Saket, Sudeep Sarkar, and Sanjukta Bhania. "Es timation of upper bound of power dis sipation in QCA circuits." IEEE transactions on nanotechnology 8.1 (2008): 116-127.

[13] Laugesen, Richard S. "New dis sipated energies for the thin fluid film equation." Communications on Pure \& Applied Analysis 4.3 (2005): 613.

[14] Bryant, Samuel J., and Benjamin B. Machta. "Energy dissipation bounds for autonomous thermodynamic cycles." Proceedings ofthe NationalAcademyof Sciences 117.7 (2020): 3478-3483.

[15] Bryant, Samuel J., and Benjamin B. Machta. "Energy Dissipation Bounds in Autonomous Thermodynamic Systems." arXiv preprint arXiv:1903.06780 (2019).

[16] Thomson, William. "9. The kinetic theory of the dissipation of energy." Proceedings of the Royal Society of Edinburgh 8(1875): 325-334.

[17] Burbury, S. H. "LII. A theorem on the dissipation of energy." The London, Edinburgh, and Dublin Philosophical Magazine and JournalofScience 13.83 (1882): 417-419.

[181 Rayleigh, Lord. "On the dissipation of energy." Van Nostrand's Eclectic Engineering Magazine (1869-1879) 12.78 (1875): 519.

[19] Thoms on, William. "2. On a Univers al Tendency in Natu reto theDis sipation of Mechanical Energy." Proceedings of the Royal Society of Edinburgh 3 (1857): 139-142.

[20] Eu, Byung C. "Kinetic theory and irreversible thermodynamics." NASA STI/Recon Technical Report A 93(1992).

[21] Bland, David Russell. The theory oflinear viscoelasticity. Courier Dover Publications, 2016. 